\title{
Criminologie
}

\section{Dangerosité et pratique criminologique en milieu adulte}

\section{Jean Dozois, Michèle Lalonde et Jean Poupart}

Volume 17, numéro 2, 1984

La dangerosité, un débat à poursuivre

URI : https://id.erudit.org/iderudit/017198ar

DOI : https://doi.org/10.7202/017198ar

Aller au sommaire du numéro

Éditeur(s)

Les Presses de l'Université de Montréal

ISSN

0316-0041 (imprimé)

1492-1367 (numérique)

Découvrir la revue

Citer cet article

Dozois, J., Lalonde, M. \& Poupart, J. (1984). Dangerosité et pratique criminologique en milieu adulte. Criminologie, 17(2), 25-51.

https://doi.org/10.7202/017198ar

\section{Résumé de l'article}

After a brief review of the criticism leveled at the definitions and use of dangerousness, this article examines how criminol-ogists work with it on a daily basis within the adult justice system.

We first describe the important place it occupies in their practice, notably in their work of evaluating and treating the clientèle. Next we analyze the process by which criminologists effect the social reconstruction of their clients' dangerousness. To do this, we ascertain how criminologists categorize the clientèle as dangerous or not dangerous, and also show the influence of the practice on the process by which criminologists define dangerousness. 


\section{DANGEROSITÉ ET PRATIQUE CRIMINOLOGIQUE}

EN MILIEU ADULTE

Jean Dozois, Jean Poupart, Michèle Lalonde*

After a brief review of the criticism leveled at the definitions and use of dangerousness, this article examines how criminologists work with it on a daily basis within the adult justice system.

We first describe the important place it occupies in their practice, notably in their work of evaluating and treating the clientèle. Next we analyze the process by which criminologists effect the social reconstruction of their clients' dangerousness. To do this, we ascertain how criminologists categorize the clientèle as dangerous or not dangerous, and also show the influence of the practice on the process by which criminologists define dangerousness.

\section{INTRODUCTION}

À l'occasion du colloque que tenait l'Association professionnelle des criminologues du Québec (A.P.C.Q.) en 1979, plusieurs praticiens s'étaient interrogés sur la notion de dangerosité. En réponse à ces interrogations et à l'invitation du comité scientifique de cette association, nous avons entrepris une recherche sur l'usage que les criminologues font (ou ne font pas) de cette notion dans leur pratique professionnelle ${ }^{1}$. Cette recherche comprend deux volets : le premier porte sur les criminologues qui travaillent au sein du système de justice pour adultes. Le second s'intéresse à ceux qui interviennent auprès des jeunes.

Nous nous proposons ici de présenter certains des résultats du premier volet de notre recherche. Mais avant, il convient de préciser davantage le contexte dans lequel s'inscrit cette recherche, puis de donner quelques précisions sur la méthodologie que nous avons utilisée.

* Jean Dozois et Jean Poupart sont professeurs à l'École de criminologie de l'Université de Montréal. Michèle Lalonde est assistante de recherche à l'École de criminologie de l'Université de Montréal.

1. Nous remercions I'A.P.C.Q. qui a financé la phase exploratoire de cette recherche ainsi que les membres de son comité scientifique de la collaboration intense qu'ils nous ont apportée. Nos remerciements s'adressent également à ceux que nous avons consultés à titre de personnes-ressources et, d'une manière spéciale, à tous les praticiens qui ont bien voulu accepter d'être interviewés. Cette recherche a pu être réalisée grâce au soutien financier de notre principal bailleur de fonds, le Conseil québécois de la recherche sociale. Cet article constitue une version révisée d'un texte paru dans les actes du colloque de l'Association professionnelle des criminologues du Québec (A.P.C.Q.) qui a eu lięu à Montréal en juin 1983 sous le thème : "Dangerosité et criminologie : fondement ou écueil d'une pratique." 


\section{LA MISE EN CAUSE DE LA NOTION DE DANGEROSITÉ}

Depuis une dizaine d'années, la notion de dangerosité fait l'objet de nombreuses critiques, notamment en criminologie, en psychiatrie et en sociologie. Ces critiques concernent et le contenu de cette notion et la légitimité de son recours.

Malgré ces critiques, la notion de dangerosité n'en continue pas moins de jouer un rôle important dans la politique pénale. On remarquera notamment l'insistance mise sur la dangerosité comme critère de décision au sujet de l'emprisonnement. Ainsi, par exemple, la Commission de réforme du droit du Canada (1976) suggérait de restreindre l'application de cette mesure aux délinquants qui, plus particulièrement, «mettent très sérieusement en danger les membres de la société», dans un but de neutralisation.

On constatera également l'adoption, en 1977, de la législation sur les «délinquants dangereux» dans le cadre du programme Paix et sécurité, lequel vise à «accroître la protection de la société contre la criminalité de violence». Encore qu'il importe de remarquer avec Landreville et Petrunik (1981) que les lois spéciales sur les délinquants dangereux semblent avoir des fonctions plus symboliques que réelles si l'on en juge par leur peu d'application.

On observera enfin l'intention de revenir à une plus grande sévérité à l'égard des délinquants présumés comme étant ou pouvant être dangereux. Songeons aux récents amendements qu'approuvait le gouvernement fédéral visant à permettre à la Commission nationale de libération conditionnelle de réincarcérer, dès leur sortie, des détenus ayant droit à la surveillance obligatoire. Ces amendements s'appliqueraient à ceux condamnés pour des crimes de violence ou de nature sexuelle, à condition cependant que les autorités démontrent que leur remise en liberté constitue un danger.

Selon Bottoms (1977), cet intérêt porté à la notion de dangerosité s'insère dans la tendance à la «bifurcation" qui s'est faite jour dans le contrôle social de la délinquance. En effet, se manifeste d'une part la volonté de réprimer rigoureusement les infractions dites de violence et d'autre part, celle de recourir à des solutions alternatives à l'emprisonnement pour la délinquance «mineure" 2 .

2. On peut se demander si, dans les faits, ces solutions ont permis une baisse substantielle de la population carcérale ou si, comme d'aucuns l'ont fait remarquer, elles n'ont pas touché des personnes qui, de toute manière, n'auraient pas été en prison. En d'autres termes, les solutions dites alternatives seraient venues se superposer à la prison et permettre une extension du contrôle social à des personnes qui n'étaient pas auparavant visées. 
En ce qui a trait aux critiques faites à l'égard de la notion de dangerosité, il faut bien dire qu'elles ne sont pas faciles à accepter et ce, pour deux raisons. En premier lieu, cette notion a occupé - et occupe toujours - une place prépondérante dans une certaine criminologie tant d'inspiration psychologique que sociologique. De la sorte, remettre en cause la dangerosité aboutit, ni plus ni moins, à remettre en cause une bonne partie du savoir criminologique. En second lieu, la notion de dangerosité est, si l'on peut dire, ancrée profondément dans les modes de penser. En effet, bien peu doutent de son existence en soit. Elle fait donc partie «des évidences" que l'on parvient difficilement à mettre en question.

Si les critiques adressées à la notion de dangerosité sont multiples, elles peuvent cependant se regrouper sous deux rubriques : celles portant sur le caractère problématique de sa définition et celles portant sur le caractère arbitraire des décisions ${ }^{3}$. Envisageons-les successivement.

Le caractère problématique de la définition de la dangerosité se trouve affirmé par la relativité de cette définition. En général, et notamment dans le domaine pénal, sont considérés comme dangereux les comportements dits de violence, c'est-à-dire les comportements portant atteinte à l'intégrité physique d'autrui. Dès lors ne serait-il pas plus justifié, comme se le demandent certains (Bottoms, 1977), de s'intéresser par exemple aux accidents de la circulation plus coûteux en termes de vies humaines que la criminalité de violence?

Davantage important est le fait que soient désignées comme dangereuses des conduites individuelles alors que des pratiques sociales affectant la santé et la sécurité des personnes telles que les mauvaises conditions de travail et la pollution ne le sont pas (Bottoms, 1977; Geis et Monahan, 1976; Shah, 1977).

Soulignons la dramatisation dont est l'objet ladite violence individuelle. Cette dramatisation, à laquelle participent entre autres les médias, s'appuie en grande partie sur les "crimes spectaculaires", crimes qui pourtant sont peu fréquents. Comme plusieurs l'ont fait remarquer, la dramatisation d'un certain type de criminalité permet d'occulter d'autres comportements préjudiciables, et plus particulièrement, ceux des classes favorisées. Elle permet également l'occultation d'autres problèmes comme ceux du chômage, de la pauvreté et des inégalités sociales en général.

3. Pour un examen plus en profondeur de ces deux types de critiques, nous nous permettons de renvoyer les lecteurs à deux articles que nous avons déjà publiés (Dozois, Lalonde, Poupart, 1981; Poupart, Lalonde, Dozois, 1982). 
Les remarques précédentes visent à souligner la relativité de la notion de dangerosité, laquelle dépend de paramètres sociaux, politiques et idéologiques, et non pas à préconiser son élargissement, c'est-à-dire son application à d'autres catégories de comportements ou d'individus. Ce risque d'un élargissement est d'autant plus présent que cette notion de dangerosité - tout comme celle de violence avec laquelle elle est d'ailleurs souvent associée - , se révèle comme facilement globalisante et extensive.

Dans le contexte de la criminologie, la dangerosité a été conçue comme l'expression ou le reflet de failles de la personnalité ou du milieu de vie. Ces failles devaient permettre d'expliquer le passage à l'acte et de différencier le délinquant du non-délinquant. À cet égard, il importe de mentionner les critiques récemment faites à la notion de personnalité criminelle, critiques ayant entre autres pour auteurs des cliniciens. Parmi ceux-ci, Debuyst $(1977,1981)$ en offre sans doute la version la plus articulée. Selon lui, la notion de personnalité criminelle conduit à isoler certains aspects de la personnalité, c'est-à-dire ses aspects essentiellement négatifs, et à négliger le contexte social dans lequel prend place le comportement. De plus, il soutient que la perspective pénale a infléchi l'examen de personnalité ${ }^{4}$ dans la mesure où elle lui pose comme objectif l'appréciation d'une personnalité en termes d'adaptation ou d'inadaptation à un certain ordre social.

Le second groupe de critiques concerne le caractère arbitraire des décisions en matière de dangerosité. Plus spécifiquement, il remet en cause la faible validité et fidélité des prévisions de la dangerosité. Cette mise en cause provient, d'une part, d'études sur les prises de décisions et d'autre part, de celles vérifiant la valeur des prédictions cliniques et statistiques.

De nombreuses études constatent que divers biais affectent les prises de décisions des professionnels. Ennis et Litwack (1974) qui ont recensé ces études soulignent que ces biais sont liés, entre autres, à la formation reçue et à la perspective adoptée par le clinicien, à sa classe sociale, au contexte dans lequel est effectué le diagnostic.

D'autres études révèlent que les prises de décisions peuvent également être marquées par différentes contingences, c'est-à-dire des considérations extérieures n'étant pas directement liées à l'objet même de la

4. On peut s'interroger sur l'avenir de l'examen de personnalité dans le système pénal dans la mesure où, de plus en plus, des textes législatifs ou administratifs définissent de manière précise et la dangerosité et les individus à considérer comme dangereux. 
décision. Ces considérations peuvent être d'ordre politique, administratif ou professionnel. Nous y reviendrons.

Mais c'est surtout à la suite d'études examinant la valeur des prédictions de la dangerosité que l'expertise professionnelle a été remise en doute ${ }^{5}$.

Ces études ont suivi pendant un certain temps des individus jugés dangereux par des spécialistes mais libérés par les tribunaux malgré ces avis. Toutes montrent que les prédictions cliniques s'avèrent souvent erronées. Elles révèlent également la tendance marquée des experts à surévaluer la dangerosité, une bonne partie des individus jugés dangereux n'adoptant pas dans les faits de comportements violents. Monahan (1978) qui a passé en revue ces travaux constate qu'au mieux, un pronostic sur trois est juste.

Qu'en est-il des pronostics effectués au moyen de méthodes statistiques? Tout comme ceux faits par les spécialistes, les prévisions statistiques de la dangerosité s'avèrent plutôt inexactes. En effet, des études ayant tenté de prédire statistiquement la dangerosité, il ressort également la tendance à surprédire la dangerosité. Selon plusieurs auteurs (Megargee, 1976; Monahan, 1976; Montandon, 1979), l'une des principales raisons expliquant l'incapacité, aussi bien statistique que clinique, de prédire la dangerosité, est la difficulté de prévoir les événements rares. L'on ne peut prédire des événements dont le taux d'occurrence est faible autrement qu'en identifiant un nombre élevé de faux-positifs, c'est-à-dire d'individus faussement évalués comme dangereux.

Outre ces études, de nombreux auteurs (Ennis et Litwack, 1974; Morris, 1974; Pfohl, 1978; Von Hirsch, 1972) dénoncent la faible valeur des prédictions cliniques et statistiques de la dangerosité et soulignent l'incapacité, dans l'état actuel des connaissances, de prévoir avec certitude les risques de danger. Quant à savoir si, dans le futur, une telle prévision sera possible, les avis sont partagés.

Toutes les critiques émises à l'égard de la notion de dangerosité démontrent l'importance d'en savoir davantage sur la façon dont les criminologues utilisent et envisagent cette notion à travers leur expérience professionnelle.

5. Pour une description détaillée de ces études, voir, entre autres, Monahan, (1978, 1981) et notre article paru dans la revue Criminologie (Poupart, Dozois, Lalonde, 1982). 


\section{MÉTHODOLOGIE}

Pour réaliser cette étude, nous avons procédé par des entretiens à tendance non directive. Cette approche nous a permis de fouiller plus en profondeur l'usage fait, au jour le jour, par les criminologues de la notion de dangerosité. D'une durée variant entre une heure et demie et trois heures, les entretiens débutaient par une consigne de départ suffisamment vaste pour laisser les interviewés libres d'aborder les thèmes qu'ils jugeaient pertinents ${ }^{6}$. Dans l'ensemble, les interviewés ont traité abondamment et avec aisance du sujet de la recherche. Ils ont parlé, entre autres, de la place de la notion de dangerosité dans les diverses facettes de leur travail et, ce faisant, de leur métier en général et du contexte institutionnel dans lequel il s'insère.

Comme il est d'usage dans les recherches de type qualitatif, notre étude n'est pas basée sur un échantillon de type probabiliste. La sélection des interviewés s'est faite de façon à tenir compte de l'importance et de la diversité des expériences de travail des criminologues. Elle s'est faite également de manière à permettre une certaine saturation des données. Nous avons ainsi interviewé trente-cinq praticiens, soit dix dans le secteur de la probation, quinze dans celui de la détention et dix dans celui des libérations conditionnelles fédérales.

Dans les services de la probation et de la libération conditionnelle, nous avons choisi les interviewés selon la répartition géographique des lieux de travail. Dans le milieu carcéral, ils ont été retenus selon le type d'institution (niveau de sécurité maximum, médium et minimum; rôle dans le réseau). Nous avons également pris en considération les diverses fonctions exercées par les criminologues ainsi que d'autres variables telles les années d'expérience, le type d'expériences antérieures et le sexe. À une exception près, tous ces praticiens œuvrent dans la région de Montréal.

\section{DE L'USAGE FAIT DE LA NOTION DE DANGEROSITÉ PAR LES CRIMINOLOGUES \\ DANS LE SYSTÈME DE JUSTICE POUR ADULTES}

Dans le cadre de ce texte, nous ne pourrons traiter de toutes les particularités relatives à chaque secteur de pratique (la probation, la détention et la libération conditionnelle). Nous tâcherons cependant de répondre aux questions suivantes : Quelle est la place et l'importance de la notion de dangerosité dans la pratique professionnelle des criminolo-

6. Cette consigne s'énonçait de la manière suivante : "J'aimerais que vous me parliez, à partir de votre expérience professionnelle, de ce qu "est pour vous la dangerosité." 
gues? Quel sens cette notion prend-t-elle selon les divers milieux de travail? Quelles critiques les criminologues formulent-ils à l'égard de l'évaluation de la dangerosité et comment en arrivent-ils à "reconstruire" la dangerosité de leur clientèle? Quelle position adoptent-ils face à l'utilisation de cette notion dans le cadre de leur travail?

\subsection{L'IMPORTANCE DE LA NOTION DE DANGEROSITÉ}

Une analyse des tâches que les criminologues ont à accomplir au sein du système pénal de même qu'une analyse des conditions d'exercice de leur métier telles qu'elles ressortent des témoignages recueillis auprès de nos interviewés, tendent à démontrer que la notion de dangerosité constitue une dimension fondamentale de leur vécu professionnel. L'importance de cette notion peut toutefois varier selon les secteurs de travail et les divers postes occupés.

La notion de dangerosité ou, en corollaire, de non-dangerosité fait partie de la pratique des criminologues de multiples façons. D’abord, elle touche directement aux deux fonctions principales que les criminologues ont à exercer à l'intérieur du système pénal : d'une part, celle de faire des évaluations de la clientèle; d'autre part, celle d'assurer le suivi de cette même clientèle. Ensuite, la dangerosité ou la non-dangerosité perçue de la clientèle affecte non seulement les attitudes que les criminologues peuvent adopter à l'égard de leurs clients mais également ce qu'ils peuvent ressentir sur un plan plus personnel dans leur métier. Enfin, la dangerosité sert à justifier les institutions dans lesquelles les criminologues travaillent.

\subsection{LA DANGEROSITÉ EN TANT QU'OBJET D'ÉVALUATION}

La dangerosité se situe d'abord au cœur des évaluations de la clientèle que les criminologues doivent produire à l'intérieur du système pénal. C'est le point de vue que soutiennent les interviewés, point de vue qui semble fondé si nous nous fions à ce qu'ils disent des directives à suivre dans la préparation et la rédaction des rapports et à la description qu'ils nous ont faite du contenu de leurs évaluations, même si le terme de dangerosité ne pourrait apparaître que peu souvent dans les rapports écrits ${ }^{7}$. Revenons sur la nature de ces évaluations dans les trois secteurs considérés.

7. Les praticiens disent employer d'autres termes tels celui de récidive violente qu'ils estiment plus précis et moins stigmatisant. Il est possible, en effet, que cette pratique puisse constituer une amélioration. Cela dit, ce n'est pas parce qu'on modifie la terminologie que l'on ne réfère pas tout de même à la notion de la dangerosité. Dire que l'on se prononce sur le risque de récidive violente ou dire que l'on se prononce sur le risque de dangerosité, sans être tout à fait la même chose, pose essentiellement le même type de problèmes et l'on peut effectivement faire les mêmes remarques à propos de la notion de violence qu'à propos de celle de dangerosité. 
Dans le secteur de la probation, les agents sont appelés à fournir deux types de rapports : les rapports d'évaluation prélibératoire destinés à la Commission québécoise des libérations conditionnelles et les rapports présentenciels demandés par la cour. La perception que les agents se font de la dangerosité diffère selon ces deux types de rapports. Pour ce qui est de la clientèle soumise à des évaluations prélibératoires, les agents estiment que, mis à part un certain nombre de "cas psychiatriques", ils ont généralement affaire à des individus peu ou pas dangereux. Utilisant des critères qui s'apparentent, semble-t-il, à ceux employés par la cour un premier délit, la moindre gravité du délit et le jeune âge du contrevenant - ils considèrent en effet que le choix même d'une mesure de probation ou d'emprisonnement de moins de deux ans est symptomatique d'une dangerosité peu élevée.

Perçue comme peu importante, la dangerosité n'en reste pas moins, indirectement, une catégorie significative dans l'appréciation de la clientèle. D'abord, elle est utilisée à la négative, pour dire que la clientèle est habituellement moins dangereuse que celle des pénitenciers ou des libérations conditionnelles fédérales. Elle sert donc de référentiel permettant de déterminer lesquels parmi les individus pris en charge par le système pénal devront être considérés aussi bien comme dangereux que comme non dangereux. Ensuite, les agents de probation tentent dans leurs évaluations formelles ou informelles d'anticiper la dangerosité future de leur clientèle. Comme le disent certains interviewés, même si le dossier est mince, il s'agit de discerner, au-delà d'un délit de moindre gravité, les signes éventuels d'un potentiel de dangerosité telles la présence de problèmes de la personnalité ou l'orientation vers des valeurs délinquantes. La dangerosité est donc une dimension marquante dans les jugements portés sur la clientèle.

Pour ce qui est des rapports présentenciels, l'appréciation de la dangerosité y occupe une place prépondérante. En effet, selon les agents, une bonne part de la clientèle représente un danger à cause des problèmes psychiatriques qu'ils éprouvent ou des crimes de violence qu'ils ont perpétrés. Plusieurs interviewés constatent un changement dans la clientèle référée par la cour. Si, auparavant, cette clientèle était constituée d'individus ayant commis des délits mineurs, actuellement, elle comprendrait également des individus qui ont posé des actes de violence, individus pour lesquels la cour s'interroge sur le bien-fondé d'une peine d'emprisonnement.

Dans le secteur de la détention, la dangerosité constitue sans doute, comme le dit un interviewé, "le facteur clé" qui préside aux multiples évaluations et décisions relatives au séjour du détenu en milieu carcéral, 
même si, dit-on, d'autres considérations semblent entrer en ligne de compte telles l'âge du contrevenant, la durée de la sentence et le nombre de places disponibles dans les institutions. Elle intervient lors du placement initial quant au choix du niveau de sécurité (minimal, moyen, maximal) et de l'institution. Elle spécifie aussi les modalités du séjour, à savoir le choix de l'unité de vie, le choix du type d'activité et le transfèrement. Elle détermine enfin l'octroi ou le refus d'une mesure prélibératoire telle l'absence temporaire avec ou sans escorte et d'une mesure de libération.

En libération conditionnelle, un certain nombre d'agents travaillent à faire "de la préparation de cas". Ils ont pour mandat de conseiller la Commission nationale des libérations conditionnelles sur le bien-fondé d'une mise en liberté. Comme le disent les agents, les évaluations sont faites en tenant compte du degré et du type de dangerosité des détenus, la Commission désirant savoir si ces derniers présentent des risques élevés de récidive et notamment, de récidive violente ${ }^{8}$. Pour illustrer l'importance de la notion de dangerosité dans les évaluations produites par les agents travaillant à la préparation des cas, soulignons ce commentaire d'un responsable de service qui, commentant des discussions internes sur l'à-propos de supprimer une telle notion, affirmait qu'une telle éventualité mettrait en cause l'essence même de leur travail.

En somme, malgré les variations existant d'un secteur à l'autre, la notion de dangerosité constitue un élément fondamental des évaluations effectuées par les criminologues et, ce faisant, de la catégorisation qui est faite des différents types de délinquants ${ }^{9}$. Dans la mesure où ces évaluations reflètent les modes d'opérer des instances de prise en charge à l'intérieur du système pénal, la notion de dangerosité apparaît ainsi comme un élément essentiel de la classification et de la répartition des délinquants.

8. Dans un contexte partiellement différent, celui de l'octroi d'une absence temporaire, voici ce que disait un agent de gestion de cas en milieu pénitentiaire relativement aux attentes de la Commission nationale des libérations conditionnelles :

"... quand un commissaire se prononce sur une absence temporaire, il veut savoir rien qu'une affaire : le gars va-t-il revenir, va-t-il faire bobo à quelqu'un, ou bien si c'est un gros big shot, va-t-il contacter ses complices pour monter un coup; c'est tout ce qu'ils veulent savoir. En quatrième lieu, bien loin derrière, le but réel de l'absence temporaire, est-ce que ça va servir à quelque chose?»

On peut cependant se demander si la perception que les agents de gestion de cas et les agents de libération conditionnelle se font des attentes de la Commission correspond effectivement aux attentes de celle-ci. Nous n'avons pas étudié directement cette question. Quoiqu'il en soit, il suffit que les agents aient de telles perceptions pour que les évaluations produites puissent en être marquées. 


\subsection{LA DANGEROSITÉ COMME OBJET D'INTERVENTION}

La notion de dangerosité affecte également les diverses interventions que les criminologues font auprès de leur clientèle. Quoiqu'il existe parmi les criminologues des divergences de vue en ce qui a trait au sens que prend et devrait prendre leur travail auprès des délinquants, notamment quant à la possibilité de faire de la réinsertion sociale dans le cadre du système pénal, ils s'entendent toutefois pour dire qu'ils reçoivent pour mission de protéger la société en assurant le contrôle de la dangerosité de leurs clients.

En pratique, il existe du point de vue des praticiens diverses mesures susceptibles d'assurer le contrôle de la dangerosité perçue de la clientèle : mesure de neutralisation par l'emprisonnement de ceux qui sont suffisamment ancrés dans une carrière criminelle pour menacer la société; mesure de "réhabilitation", de "resocialisation" ou de "réinsertion sociale" par le «counselling» ou par des actions de soutien telles l'aide à la recherche d'emploi; mesure de surveillance de la conduite des délinquants de manière à réduire les risques de violence ou d'évasion à l'intérieur du contexte pénitentiaire ${ }^{10}$ et de manière à diminuer les risques de récidive dans les contextes de la probation et de la libération conditionnelle.

La plupart des praticiens espèreraient mettre l'accent sur la réinsertion sociale des délinquants ${ }^{11}$, mais ils estiment que leur travail consiste

9. Le terme de délinquant porte à confusion et à controverse depuis quelques années en criminologie. Qu'il soit entendu qu'il sera employé tout au long de ce texte pour désigner les individus, jeunes ou adultes qui, parce qu'ils sont présumés avoir adopté un comportement défini comme non conforme aux lois, sont pris en charge par le système pénal et, à ce titre, reçoivent le statut de délinquant mineur ou adulte. Cette acception plus générale du terme se situe dans le courant actuel de la criminologie qui voit un lien entre l'action des institutions de contrôle social et la délinquance comme produit socialement fabriqué. Cette définition ne correspond toutefois pas au sens habituel que l'on donne à ce terme dans le contexte nord-américain où l'on réserve cette étiquette pour désigner le seul groupe des délinquants mineurs.

10. On peut interpréter les diverses mesures telles que le "counselling", les sorties, les visites et les divers programmes à l'intérieur des institutions comme autant de moyens d'améliorer le sort des détenus. Sans mettre en cause la bonne volonté des gens chargés de l'application de telles mesures, on pourrait également se demander si ces divers moyens, en rendant la prison plus viable, ne répondent pas à des objectifs plus cachés et fondamentaux liés au bon fonctionnement et à la survie des institutions. Voir à cette effet l'excellent article de Jean François (1979) et l'étude classique de Goffman (1968) sur l'asile.

11. Ce modèle d'intervention professionnelle avec ses quelques variantes correspond d'ailleurs à l'idéologie dominante en criminologie où l'on tente d'enrayer l'agir du délinquant par des interventions au niveau de sa personnalité, de ses valeurs et, plus rarement, de son milieu, réduisant ainsi sa dangerosité. 
surtout à exercer un certain contrôle sur la clientèle ${ }^{12}$. Prenons l'exemple des libérations conditionnelles.

Les agents assignés à "la surveillance des cas" ont pour mandat, c'est du moins la perception qu'ils en ont, d'empêcher la récidive de leur clients et, en particulier, de ceux qui présentent des risques élevés de dangerosité «en raison du caractère violent de leur délit». Ils estiment en effet que, sans que cela soit nécessairement le seul objectif que les autorités leur fixent, le contrôle de la dangerosité n'en reste pas moins une dimension prioritaire de leur travail. "La surveillance des cas", expression en soi significative, s'effectue d'ailleurs en conséquence. Les agents sont tenus d'avoir des rencontres plus fréquentes avec les cas jugés dangereux. Ils doivent régulièrement faire rapport de la progression des cas et s'assurer que les libérés respectent les conditions particulières de leur mise en liberté telles que se présenter à la police, participer à un programme de réhabilitation ou s'abstenir de fréquenter certains territoires, certains établissements ou certaines personnes. Les agents se trouvent ainsi placés en situation d'autorité par rapport aux libérés puisqu'ils ont le pouvoir et le devoir de suspendre la libération si ces derniers ne se soumettent pas à ces conditions ${ }^{13}$.

La surveillance des cas s'effectue également de manière plus indirecte par les renseignements que les agents peuvent recueillir auprès des libérés, de leur famille et de leurs amis ou encore auprès des employeurs et de la police. Les agents ne pouvant pas être, comme le disent certains interviewés, vingt-quatre heures sur vingt-quatre auprès de leurs clients ni même assurer un contrôle serré de tous les cas, ils concentrent leurs énergies sur un petit nombre d'entre eux, certains jugés plus intéressants parce que plus coopératifs, d'autres jugés plus dangereux soit en raison de leur dossier, soit en raison de l'imminence d'une récidive. Pour dépister ceux qui sont sur la voie d'une récidive, les agents se basent, entre autres, sur ce qu'ils appellent les indices de désorganisation : la perte d'emploi,

12. Dans leur discours sur le sens à donner ou le sens que prend leurs interventions, le thème de la relation d'aide versus de contrôle revient avec insistance et constitue sans nul doute une préoccupation majeure des praticiens. La majorité d'entre eux d'ailleurs trouvent difficilement conciliables ce double objectif. Toutefois, certains criminologues tentent de rendre compatibles ces deux objectifs. Ainsi, un agent de libération conditionnelle peut décider de suspendre un libéré et de le replacer en institution en estimant que cette mesure contraignante est de nature à faire réfléchir son client et par voie de conséquence, à favoriser ultimement sa réintégration dans la société. De la même façon, un agent peut considérer le fait pour un libéré conditionnel de se soumettre aux conditions de sa libération comme le signe de sa capacité à se soumettre aux exigences de la vie en société. Certains praticiens tentent donc de réconcilier mesure d'aide et mesure de contrôle.

13. En pratique cependant, l'agent possède une certaine marge de discrétion dans l'application des règlements. 
l'absentéisme au travail, les fréquentations douteuses, les problèmes familiaux ou sentimentaux, l'attitude plus ou moins agressive du libéré en entrevue ou le non-respect des conditions de la libération. Pour les agents, ces signes sont révélateurs d'un malaise chez le libéré, malaise qui pourrait le conduire à la récidive. Notons que ces indices mettent l'accent sur la conformité sociale dans l'appréciation des comportements. De plus comme le mentionnent certaines études ${ }^{14}$, les détenus et les libérés étant conscients des attentes des agents, cela ouvre ainsi la porte à de multiples tractations entre les deux partis et rend plus difficile que s'établisse entre eux une véritable relation de confiance.

Le contrôle de la dangerosité structure donc les interventions des criminologues même s'il ne représente pas ce que la plupart souhaiteraient privilégier.

\subsection{LA DANGEROSITÉ COMME OBJET DE PRÉOCCUPATION \\ DANS LE CADRE DE LA RELATION PRATICIEN-CLIENT}

La dangerosité touche également à ce que peuvent ressentir les criminologues dans le cadre de leurs relations avec les clients. Plusieurs de nos interviewés ont parlé de la dangerosité dont ils pouvaient être l'objet dans l'exercice de leur métier compte tenu des particularités de la clientèle et de son milieu d'origine, et du contexte dans lequel s'insère leur pratique. Bien qu'elle n'ait pas été abordée par tous nos interviewés, cette facette de la dangerosité revient avec suffisamment d'insistance au cours de certains entretiens pour laisser croire qu'elle représente une composante importante de la pratique. Vécue différemment par chacun, elle se manifeste aussi bien chez les praticiens de sexe masculin que féminin, aussi bien chez les praticiens plus jeunes que plus âgés. Certains de nos interviewés nous en parlent comme d'un stress sous-jacent à la pratique qui, sans être dramatique, ne s'avère pas moins un aspect préoccupant du métier.

Cette crainte d'être l'objet d'attaque de la part de la clientèle se retrouve dans les trois secteurs de pratique. En probation, elle apparait comme une préoccupation centrale pour les practiciens qui ont pour tâche de faire des évaluations ou du suivi auprès de la clientèle jugée aux prises avec des problèmes psychiatriques. Ces cas apparaissent aux yeux de certains agents comme souvent «imprévisibles et incontrôlables» et suscitent à ce titre diverses inquiétudes : celle que l'individu entre en crise durant l'entrevue; celle qu'il s'en prenne à l'agent lors d'une évaluation

14. Sur la perception que pensent se faire les libérés conditionnels des attentes de leurs agents, voir l'article de C. Arsenault (1981). 
négative ou face aux frustrations engendrées par les contraintes d'une mesure de probation. À cet égard, certains agents se plaignent d'une part d'être mal protégés et d'autre part d'être mal outillés pour faire face à ces cas étant donné leur manque de formation et le peu de ressources disponibles.

Cette dimension se fait également sentir dans le secteur de détention, "véritable laboratoire de situations dangereuses» pour reprendre les termes d'un interviewé. Par les contraintes qu il fait vivre et, par les tensions qu'engendre la sous-culture carcérale, le milieu pénitentiaire est perçu comme un lieu à risques élevés pour les détenus mais également pour le personnel : risque de violence verbale ou physique durant une entrevue, risque de se trouver au mauvais endroit au moment d'une prise d'otage ou d'un règlement de compte, risque de représailles à l'extérieur.

On retrouve des préoccupations semblables dans le secteur des libérations conditionnelles où les agents craignent parfois des représailles de certains de leurs clients ou de leur milieu d'appartenance. Quelques agents nous ont ainsi mentionné qu'il n'était pas toujours facile d'effectuer des visites à domicile où «l'on se sent comme un corps étranger" tant par rapport au libéré que par rapport à sa famille, laquelle est jugée souvent tout aussi hostile.

En pratique, cependant, les agents disent que les manifestations réellès de violence envers le praticien sont plutôt rares. En outre, les situations où l'individu risque d'être violent seraient relativement prévisibles ( «tu apprends avec l'expérience à savoir quand il va se passer quelque chose dans une aile»; "tu le vois dans la face du gars quand il est sur le bord de faire un acting out") et en partie désamorçables par l'attitude même du praticien. Sans vouloir exagérer l'importance de cette dimension dans la pratique des criminologues, il est facile d'imaginer, quoique difficile à estimer, que l'insécurité ressentie par les praticiens puisse affecter leurs perceptions et attitudes à l'égard des clients.

\subsection{LA DANGEROSITÉ ET LA LÉGITIMATION DE LA PRISON}

À un niveau moins explicite, la dangerosité peut servir également à légitimer les institutions dans lesquelles les criminologues travaillent et le choix des mesures qu'ils sont amenés à prendre. Examinons, par exemple, la position qu'ils adoptent à l'égard de la prison.

Tant en probation et en libération conditionnelle que dans le secteur de la détention, les praticiens se montrent très critiques par rapport aux effets de la prison. Ils reprennent les reproches habituels faits à son endroit : la prison détériore, stigmatise, fait vivre des tensions, provoque 
le désespoir, initie aux modes de vie criminelle. En outre, très peu croient que la prison puisse servir à réhabiliter. Malgré cela, plusieurs n'en considèrent pas moins que la prison est nécessaire, ne serait-ce que pour la neutralisation d'un groupe restreint d'individus dangereux ${ }^{15}$. La dangerosité sert donc d'argument majeur pour justifier une mesure à laquelle on souhaiterait, du moins en principe, ne pas avoir à recourir (de même que l'absence de dangerosité sert en contre-partie à justifier le recours à des mesures plus souples comme celle des travaux communautaires).

\section{LE SENS ATTRIBUÉ À LA NOTION DE DANGEROSITÉ}

Avant d'aborder le sens attribué à la notion de dangerosité, notons que les praticiens nous parlent de cette notion non pas en des termes scientifiques et théoriques tels ceux de l'état dangereux développés par Pinatel mais comme d'une catégorie pratique qu'ils utilisent au jour le jour dans leur travail. Ceci dit, ils mentionnent qu'ils définissent la dangerosité à la fois dans des termes semblables à ceux du sens commun - adopter un comportement violent à l'égard de soi et surtout des autres - et à la fois selon le sens qu'en donnent les autorités - - par exemple la récidive de nature violente - même si certains d'entre eux trouvent cette définition trop restrictive. De plus, la signification qu'ils donnent à cette notion reflète les impératifs particuliers de leur milieu de travail. Ce dernier demande un plus ample développement.

Dans les secteurs de la probation et des libérations conditionnelles, les agents sont surtout centrés sur l'évaluation de la "dangerosité sociale», soit sur l'évaluation des risques éventuels de récidive violente. La situation semble partiellement différente dans le secteur de la détention. La dangerosité sociale cède le pas à la "dangerosité carcérale", soit à l'évaluation du danger que le détenu peut présenter pour le bon fonctionnement et la survie de l'institution. En d'autres mots, les agents et, peut-on le supposer, le personnel dans son ensemble, sont moins préoccupés par les torts que l'individu pourrait causer à la société que par les torts qu'il pourrait causer à l'intérieur même du milieu carcéral.

Dans le contexte pénitentiaire, sont ainsi considérés dangereux : 1) ceux que l'on estime présenter des risques de violence à l"égard des autres détenus ou du personnel, violence que l'on attribue soit à des problèmes de personnalité, soit à l'incapacité de supporter la détention, soit à la participation à certaines activités illicites à l'intérieur du milieu carcéral;

15. Certains praticiens pensent aussi que la prison est un moyen pour décourager de la récidive ou encore, un dernier recours pour ceux qui, par exemple dans le contexte des libérations conditionnelles, refusent de coopérer. 
2) ceux qui risquent de s'évader et d'être violents pendant leur évasion ${ }^{16}$;

3) ceux qui enfin risquent de se suicider ou de s'automutiler face aux frustrations de la vie carcérale. À ces diverses catégories d'individus jugés dangereux, s’ajoute encore le groupe de détenus que l'on estime comme n'étant pas dangereux mais comme pouvant être l'objet de la dangerosité des autres détenus en raison de règlements de compte, d'une réputation de délateurs ou de délits impopulaires tels ceux de nature sexuelle (par exemple, la pédophilie). Pour assurer leur sécurité, ces détenus sont souvent confinés dans les quartiers de protection ou de ségrégation.

Pour illustrer la différence faite entre la dangerosité sociale et la dangerosité carcérale, les agents distinguent parfois les détenus qui sont dangereux à l'extérieur de ceux qui sont dangereux à l'intérieur de la prison. Ainsi, le chef de la mafia ou le pédophile fonctionneraient relativement bien à l'intérieur des murs alors qu'ils constituent une menace à l'extérieur. À l'inverse, certains détenus, auteurs de délits mineurs, acceptent mal de faire du temps et à ce titre, présentent un potentiel de dangerosité.

Dans le contexte pénitentiaire, la dangerosité sociale se distingue donc de la dangerosité carcérale, celles-ci se superposant plus ou moins selon les divers moments de l'incarcération. Ainsi à l'entrée, on tente d'apprécier la conduite future du détenu à l'intérieur des murs en tenant compte d'une part de sa dangerosité sociale telle qu'elle peut se manifester par la nature de son délit et d'autre part, de sa dangerosité carcérale, c'est-à-dire en tenant compte de l'image que l'on peut se faire du comportement habituel de ce type de détenu à l'intérieur de l'institution. Sont de la sorte classés dans les institutions à sécurité maximale ceux que l'on estime présenter des risques de violence ou d'évasion ou encore, ceux qui risquent d'être victimes de violence. Se retrouvent dans les institutions à sécurité moyenne ou minimale ceux pour qui ces risques sont évalués moindres. À la fin de la période d'incarcération, au moment où l'on s'interroge sur la dangerosité sociale du détenu une fois qu'il sera remis en liberté, celle-ci devient à son tour éclairée par la dangerosité carcérale dans la mesure où l'on tient compte de sa conduite institutionnelle. Les praticiens insistent toutefois sur la relativité d'un tel critère en raison des conditions particulières de la détention. Reste que dans le jugement que l'on porte sur lui, le détenu traîne, en plus de son passé judiciaire, tout son passé institutionnel.

16. En milieu pénitentiaire, on établit la distinction entre le danger d'évasion et le danger en évasion. 


\section{LES CRIMINOLOGUES ET L'ÉVALUATION DE LA DANGEROSITÉ}

Dans cette section, nous allons tenter de synthétiser les principales critiques faites par nos interviewés concernant l'évaluation de la dangerosité et de démontrer comment cette évaluation, loin d'être objective, met en branle un processus de reconstruction sociale où s'entremêlent aussi bien les stéréotypes des praticiens à propos de la délinquance que des considérations liées aux circonstances particulières dans lesquelles se prennent les décisions.

\subsection{LES CRITIQUES RELATIVES À L'ÉVALUATION DE LA DANGEROSITÉ}

De nombreuses réserves ont été formulées par les criminologues interviewés à l'égard de l'évaluation de la dangerosité. Ils estiment que la dangerosité est difficile à prédire et ce, pour de multiples raisons. Premièrement, elle leur apparaît comme une notion "floue", "vague", "difficile à cerner", "utilisée à toutes les sauces" même s'ils s'entendent généralement pour dire qu'elle s'emploie dans le sens de violence physique. L'ambiguïté au niveau même de sa définition compliquerait d'autant les évaluations.

Deuxièmement, ils considèrent qu'il n'existe pas d'instruments sûrs pour la mesurer. La formation universitaire a beau servir d'arrière-plan, les tests appris ne s'appliquent pas ou peu. Comme le disent certains, l'évaluation de la dangerosité est une question de "feelings" et d'expérience; elle est une "affaire de jugement» même s'il est póssible de se référer à un certain nombre de critères (âge, délit, antécédents, comportement institutionnel, etc.). Ceux-ci d'ailleurs sont relatifs. Ainsi en va-t-iI de la violence démontrée en cours de détention qui sert souvent de point de référence pour juger de la dangerosité future du détenu. Les praticiens estiment que ce critère doit être pondéré puisque cette violence peut tout autant être imputable aux conditions de vie en prison.

Troisièmement, la dangerosité est également difficile à prédire, affirment certains praticiens, "parce qu'on travaille avec du matériel humain". L'individu peut changer. Ce n'est pas parce qu'on l'a déclaré un jour dangereux, jugement constituant «un instantané», "une sorte de cliché pris à un moment donné de sa vie», qu'il le restera forcément pour le restant de sa vie. En outre, les probabilités d'adopter un comportement violent dans le futur sont tout autant liées aux situations qu 'il sera amené à vivre, situations en elles-mêmes imprévisibles. Il se peut que l'individu ne soit plus jamais placé dans des situations analogues à celles l'ayant amené à commettre son délit (c'est le cas, par exemple, du meurtre passionnel). Il se peut également que certaines circonstances de sa vie 
telles se trouver un emploi ou avoir une relation stable l'amènent à mieux réagir aux situations.

Quatrièmement, l'évaluation de la dangerosité serait sujette à la subjectivité des praticiens de même qu'elle serait tributaire du contexte social dans lequel se prennent les décisions. Ainsi, souligne un interviewé, il est possible qu'un praticien issu d'un milieu dévaforisé se laisse moins impressionner par une bataille de taverne. Il est possible également, mentionne un autre interviewé, que le cas jugé grave dans une communauté rurale passe pour un cas bénin ailleurs ${ }^{17}$.

La dangerosité n'est pas que difficile à prédire. Pour les praticiens, elle est préjudiciable à ceux qui en portent l'étiquette. En un mot, elle stigmatise. Il faut donc se méfier de son utilisation dans les rapports puisque, qu'on le veuille ou non, "cela impressionne et risque d'affecter la manière d'aborder les cas".

Suite à ces diverses critiques formulées à l'endroit de l'évaluation de la dangerosité, se pose la question de savoir comment les praticiens en arrivent concrètement à se prononcer sur quelque chose qu'ils estiment flou, vague, subjectif et sujet à diverses interprétations. S’il s’agit, comme le disent certains, "d'une affaire de jugement", de quelle façon en viennent-ils au jour le jour à porter un tel jugement?

\subsection{L'ÉVALUATION DE LA DANGEROSITÉ \\ COMME PROCESSUS DE RECONSTRUCTION}

L'évaluation de la dangerosité telle qu'elle s'élabore à travers les multiples étapes de la prise de décision, constitue un processus de reconstruction sociale dans lequel interviennent les perceptions des agents relativement aux délinquants dangereux et non dangereux et des considérations pratiques liées à cette prise de décision. Dans cette reconstruction intervient d'une part la manière de travailler des praticiens - les représentations qu'ils se font dans leur milieu de travail des individus dangereux et non dangereux, leurs façons d'interpréter et de négocier les cas, les considérations de carrière ou administratives sousjacentes aux recommandations. Interviennent d'autre part les circonstances sociales et politiques entourant les évaluations - par exemple, un contexte politique plus favorable ou moins favorable à des mesures plus libérales à l’égard des délinquants.

17. Malgré ces critiques qui tendent toutes à démontrer que les évaluations sont peu sûres, plusieurs praticiens estiment néanmoins qu'ils ont une certaine compétence ou encore, qu'ils sont parmi les professionnels qui ont le plus de compétence pour se prononcer sur cette question. 
Dire que la dangerosité est un construit social, c'est dire qu'elle n'est pas une qualité que possèdent en soi certains actes ou certains individus. Plutôt, elle est une qualification sociale apposée à certains actes et pas à d'autres de même qu'à certains individus et pas à d'autres. En premier lieu, l'évaluation de la dangerosité présuppose une certaine définition des conduites qui, dans un contexte social donné, seront considérées dangereuses. Dans la section précédente, nous avons vu que la définition que les praticiens donnent à la notion de dangerosité s'articule surtout autour de l'idée de la violence, notion elle-même ambiguë, et que cette définition a tendance à prendre une connotation particulière selon les impératifs propres à chacun des milieux de travail. Il suffirait que les présupposés à la base de cette définition se modifient pour que la représentation des individus ou des groupes sociaux considérés comme dangereux s'en trouve profondément modifiée, avec les changements de clientèle que cela pourrait entraîner à l'intérieur du système pénal. Tel serait le cas si nous entendions par dangerosité les conduites individuelles ou collectives causant des préjudices à une société telles l'évasion fiscale ou les préjugés sociaux.

En second lieu, l'évaluation de la dangerosité implique un processus d'attribution par lequel on assigne à certains individus le statut de dangereux (ou de non-dangereux). Dans le contexte pénal, ce processus d'attribution s'effectue par l'intermédiaire d'une série d'agents sociaux dont, entre autres, les criminologues.

Dans la suite du texte, nous traiterons de trois aspects de la reconstruction faite de la dangerosité par les praticiens : l'importance du savoir pratique; le modèle sous-jacent à la catégorisation de la clientèle; les considérations extérieures liées à la prise de décision. Nous illustrerons ces aspects à partir principalement de l'exemple du secteur des libérations conditionnelles.

\subsection{L'IMPORTANCE DU SAVOIR PRATIQUE}

L'évaluation de la dangerosité se réfère en grande partie à un savoir pratique, c'est-à-dire à un savoir fondé sur l'expérience acquise sur le terrain, "sur le plancher des vaches" pour reprendre lexpression d'un interviewé. Cela ne signifie pas que ce savoir soit sans aucune relation avec la formation universitaire. Cela signifie plutôt que les praticiens apprennent par et dans la pratique des modes d'opérer par rapport aux situations et aux problèmes concrets qu'ils rencontrent quotidiennement dans l'exercice de leurs fonctions. 
Des contacts quotidiens avec la clientèle, les collègues, les autres intervenants et la police, des relations avec les autorités décisionnelles, des tentatives faites auprès de la clientèle (essajs et erreurs), les praticiens en arrivent à "se faire une idée" du type de clients qu'ils desservent : leurs caractéristqiues sociales et le genre de problèmes qu’ils éprouvent. Ils se font également une idée des solutions à adopter ainsi que de la façon de les négocier tant auprès des organismes concernés ( «c'est notre business de savoir comment négocier avec la Commission nationale des libérations conditionnelles") que des clients eux-mêmes ( «un vieux routier sait à quoi s'attendre lorsque tu le rencontres").

En somme, partant des impératifs de la pratique - les choses à faire et à ne pas faire dans telles ou telles circonstances - et des images véhiculées dans le milieu concernant les individus qui sont ou ne sont pas dangereux, les praticiens apprennent à catégoriser leur clientèle, à se constituer un système de classement servant de point de repère dans la prise de décision. Ainsi, toute la clientèle n'est pas perçue comme en soi dangereuse ni comme dangereuse de la même façon.

Il ne faudrait pas croire cependant qu'une prise de décision, fondée sur un système de catégorisation, est un processus statique où les praticiens appliquent mécaniquement des catégories. Il s'agit plutôt d'un processus dynamique. D une part, la manière de procéder varie d'un cas à l'autre même si une certaine routine peut s*installer. Cette variation s'explique, entre autres, par la négociation existant entre le praticien, son client, les autres intervenants et les autorités. D'autre part, le système de catégorisation lui-même peut se modifier suite, par exemple, à des changements survenus dans la clientèle comme cela semble s'être produit en probation dans le cadre des rapports présentenciels.

\subsection{LES COMPOSANTES DE LA CATÉGORISATION DE LA CLIENTÈLE EN INDIVIDUS DANGEREUX ET NON DANGEREUX}

À partir de quoi les praticiens caractérisent-ils les individus en dangereux et non dangereux? Nous tenterons ici de dégager les composantes fondamentales de la catégorisation de la clientèle telles qu'elles se dégagent des témoignages recuejllis. Ces composantes traduisent les représentations que se font les praticiens de la clientèle. Il importe de souligner que ces représentations ne sont pas à confondre avec la réalité. Comme nous l'avons mentionné précédemment, les comportements et les individus ne sont pas en soi dangereux, quoique l'on puisse penser spontanément le contraire, mais ils le sont parce qu'on leur en confère socialement le statut. Marquant la pratique et marquées par elle, les représentations constituent diverses manières de concevoir une réalité, en 
l'occurrence la dangerosité ou la non-dangerosité de la clientèle prise en charge par le système pénal.

Partant des représentations que les praticiens se font de la dangerosité, nous pouvons ainsi distinguer trois composantes de la catégorisation de la clientèle : 1) la nature du délit et les antécédents; 2) le type de problème à l'origine du délit ou du comportement violent; 3) l'aptitude et la volonté qu'a l'individu de changer.

Aux yeux des praticiens, la nature du délit et les antécédents constituent des éléments majeurs dans la classification de la clientèle sur un continuum entre dangerosité et absence de dangerosité. Sont perçus dangereux ou pouvant être dangereux ceux qui ont commis des délits dits de violence contre la personne : meurtre, agression sexuelle, vol à main armée, voie de fait, séquestration ou prise d'otage. A contrario, sont vus comme non ou moins dangereux ceux dont les délits ne sont pas considérés comme violents tels la fraude ou la prostitution.

Du point de vue des praticiens, les antécédents qualifient davantage la nature du délit. Si le fait d'avoir commis un délit violent manifeste déjà un potentiel de violence, le fait d'en avoir commis plusieurs révèle une plus grande dangerosité. Selon eux, l'aspect répétitif, surtout s'il y a aggravation dans la nature des délits, témoigne soit d'une intentionnalité méchante de la part du délinquant, le délit ne pouvant plus être mis au compte d'un accident de parcours, soit d'une détérioration de ses problèmes.

Bien que les praticiens estiment que le délit et les antécédents s'avèrent des points de repère généraux dans l'assignation de la dangerosité d'un individu, ils trouvent toutefois ces critères insuffisants. Ainsi, ils considèrent qu'un délit mineur peut cacher des problèmes majeurs chez l'individu, lesquels pourraient résulter en une criminalité plus dangereuse. C'est le cas notamment des délinquants qui sont perçus comme ayant des problèmes psychiatriques et dont la situation, croient-ils, pourrait se détériorer encore davantage. C'est le cas également des «petits délinquants" qui semblent s'orienter vers une carrière criminelle et qui, à ce titre, peuvent devenir plus menaçants pour la société. À l'inverse, un délit grave ne signifie pas nécessairement, disent-ils, que l'individu restera indubitablement dangereux. Des praticiens citent l'exemple de quelqu'un ayant commis un crime passionnel, lequel, étant lié à des circonstances particulières, aurait moins de chance de se reproduire.

De l'avis des praticiens, il en va de même pour les antécédents. Qu'un individu n'ait pas d'antécédents ne veut pas dire qu'il ne pourrait pas être dangereux et inversement, qu'il ait des antécédents, ne signifie 
pas nécessairement qu'il pourrait être dangereux. Ainsi, il se peut qu'en raison d'un âge plus avancé, un détenu désire se ranger et éviter de retourner en prison, diminuant par le fait même sa dangerosité.

Nous touchons ici à la seconde composante de la catégorisation de la clientèle : l'évaluation du problème que les praticiens estiment être à la base du délit ou du comportement violent. À cet égard, la question de la présence ou de l'absence de contrôle est capitale dans l'appréciation de la dangerosité. Sont d'abord perçus dangereux ceux qui font preuve, semble-t-il, d'une absence totale ou partielle de contrôle, à cause de problèmes psychiatriques ou de la personnalité. Les cas psychiatriques seraient dangereux parce que, dit-on, ils sont imprévisibles et incontrôlables. Certains praticiens comparent ces cas psychiatriques «imprévisibles et incontrôlables» au voleur à main armée «manipulateur mais qu'on voit venir de cent milles à la ronde».

Selon eux, sont également dangereux les délinquants qui font preuve de contrôle mais qui utilisent ce contrôle à des fins criminelles. Entrent dans cette catégorie ceux qui, pensent-ils, ont opté résolument pour un mode de vie criminel tels les membres de la mafia, y inclus leurs chefs qui font exécuter leurs crimes par les autres. La dangerosité de cette dernière catégorie se caractérisait par un refus systématique des normes sociales. À l'inverse, sont réputés moins dangereux ceux possèdant un meilleur contrôle d'eux-mêmes ou encore, ceux moins enracinés dans une carrière criminelle. Il faut ajouter cependant que cette distinction faite par les praticiens entre les délinquants qui souffrent d'un manque de contrôle et ceux qui utilisent mal leur contrôle, n'est pas toujours très tranchée, les deux pouvant se retrouver simultanément dans la représentation qu'ils se font de certains clients.

La compréhension du "problème qui est à la source du délit» ne suffirait pas toutefois pour juger de la dangerosité du délinquant. Encore faut-il évaluer d'une part l'aptitude du délinquant à changer et d'autre part, la volonté qu'il a d'y parvenir, évaluation qui s'avère la troisième composante de la catégorisation de la clientèle. Selon les praticiens, le délinquant aura d'autant plus de chances de s'en sortir et par le fait même d'atténuer sa dangerosité, qu'il a, soit les moyens de régler ses problèmes, soit le désir de modifier sensiblement sa conduite, ou les deux.

Ainsi, dans le cas où la délinquance est imputée à des problèmes psychiatriques ou à des problèmes de la personnalité, le pronostic serait moins favorable à l'égard des premiers, lesquels sont considérés comme plus handicapés. Référant aux cas psychiatriques, une interviewée déclarait à ce propos qu'un individu n'avait pas réglé son problème du seul 
fait qu'il en prenait conscience ou qu'il se montrait soucieux de le régler. Il fallait également qu'il en ait la capacité.

Il y aurait donc les délinquants désireux de s'en sortir mais qui en possèdent plus ou moins les moyens et inversement, ceux qui, même s’ils en possèdent les moyens, ne veulent pas s'en sortir. Certains agents de libération conditionnelle citent l'exemple des détenus placés en surveillance obligatoire qui, "même s'il ne faut pas généraliser, ne veulent rien savoir». Ils donnent également l'exemple des délinquants qui, acoquinés avec le milieu criminel, font mine de se repentir et se jouent du système en faisant semblant d'adopter une bonne conduite.

En pratique cependant, même pour le délinquant qui veut s'en sortir et qui en possède jusqu'à un certain point les moyens, les praticiens estiment que la chose n'est pas simple. L'individu peut être bien intentionné mais éprouver au moment de sa réinsertion sociale des difficultés comme se trouver un emploi ou réintégrer le milieu familial. Cela le rend d'autant plus vulnérable aux tentations s'il se trouve, dit-on, dans un milieu social où ces tentations sont nombreuses.

Comment les agents décèlent-ils cette capacité et cette volonté de changer de la part du détenu ou du libéré? C'est au moyen d'un décodage des attitudes et des comportements adoptés par le passé, notamment en institution, ou durant les entrevues que les agents se font une opinion sur ce point. Ainsi, dans le cadre d'une mesure de libération conditionnelle, les agents tentent, à partir de l'étude du dossier, des avis qu'ils reçoivent et de leurs propres contacts avec le détenu, de se faire une idée de la conduite future de ce dernier en soulevant les questions suivantes : 1) le détenu a-t-il réglé ses problèmes? S'il se montre agressif en entrevue, ou s'il a adopté un comportement violent à l'intérieur de l'institution même s'il faut relativiser cette violence compte tenu des conditions de la détention - , ne serait-ce pas le signe que le détenu est encore incapable de faire face à la frustration et de se plier aux exigences de la vie en société? 2) Le détenu regrette-t-il ses actes passés? 3) Est-il sincère lorsqu'il prend des résolutions? Il est possible de se guider en partie sur le comportement adopté à l'intérieur des institutions carcérales, par exemple sur sa participation aux différents programmes, mais cette bonne conduite du détenu à l'intérieur des murs est-elle la manifestation d'un véritable changement chez l'individu ou un conformisme utilisé dans le but de manipuler les autorités? 4) Enfin, le détenu est-il réaliste dans ses projets de sortie, par exemple lorsqu'il affirme qu'il a d'excellentes chances de se trouver tel emploi ou de retourner vivre avec sa famille? Autant de questions qui, sans être posées toujours d'une manière systématique, guident les praticiens dans leur appréciation. 
En résumé, l'évaluation de la dangerosité et la catégorisation de la clientèle en individus dangereux et non dangereux est un processus complexe où s'entremêlent les idées que les praticiens se font, partant de la pratique, de la nature de la délinquance, des problèmes à son origine et des possibilités de changement. Il est à remarquer que cette façon d'envisager la délinquance correspond à une criminologie d'inspiration positiviste.

\subsection{L'ÉVALUATION DE LA DANGEROSITÉ COMME PROCESSUS SOCIALEMENT NÉGOCIÉ ET POLITIQUEMENT CIRCONSCRIT}

Troisième aspect de la reconstruction sociale de la dangerosité : interviennent dans la prise de décision des praticiens, des considérations dépassant leur stricte perception des caractéristiques des délinquants. Ces considérations touchent au statut des professionnels dans leur milieu de travail et aux circonstances sociales et politiques dans lesquelles se prennent les décisions. L'évaluation de la dangerosité à travers les multiples étapes de la prise de décision est un processus négocié dans lequel entrent en ligne de compte les impératifs de carrière telle la crédibilité des professionnels, les multiples tractations et jeux de pouvoir entre le pratjcien et son client et entre les différents groupes d'agents à l'intérieur du système pénal, et les enjeux politiques telle la bonne réputation des institutions pénales.

Bien qu'il y aurait beaucoup à dire sur cette dimension, nous nous contenterons d'apporter ici une illustration de la manière dont la prise de décision des praticiens peut être affectée par l'appréciation des conséquences professionnelles, sociales et politiques des recommandations qu'ils peuvent faire. Plusieurs agents nous ont mentionné comment, à la suite d'une récidive spectaculaire, la Commission nationale des libérations conditionnelles et eux-mêmes se montraient plus réticents, du moins pendant un certain temps, à octroyer une mesure de libération pour la catégorie de détenus concernés par ce type de récidive. Une récidive spectaculaire met donc en cause et la réputation du service des libérations conditionnelles qui peut être accusé, ou avoir peur d'être accusé, d'avoir fait preuve de trop de laxisme, et la réputation de l'agent qui peut être accusé ou se sentir accusé d'avoir mal accompli son travail. Un praticien racontait comment un collègue avait senti sa carrière compromise à la suite d'une telle récidive spectaculaire.

À en juger par les témoignages recueillis, les agents tiennent à la fois compte, dans l'appréciation des cas, des conséquences sociales d'une récidive éventuelle et des répercussions de cette récidive sur leur carrière. La prise en considération de ces répercussions pourrait expliquer l'atti- 
tude conservatrice que semblent adopter les praticiens dans leurs recommandations. Un interviewé racontait ainsi qu'à peine le tiers des cas examinés faisaient l'objet d'une recommandation de mise en liberté. Si tel était le cas, cette attitude relativement conservatrice pourrait refléter que les agents préfèrent ne pas prendre des chances dans les cas incertains bien que d'autres éléments pourraient intervenir comme l'assimilation des attentes de la commission ou un resserrement des politiques.

\section{LES CRIMINOLOGUES FACE À LA NOTION DE DANGEROSITÉ}

Dans cette section, nous tracerons un portrait d'ensemble de la position qu'adoptent les criminologues face à la notion de dangerosité. Nous avons déjà vu que les praticiens formulent de nombreuses critiques à l'égard de son évaluation. Malgré ces critiques, la plupart des praticiens considèrent important de se prononcer sur la dangerosité. Certains estiment que c'est une question de responsabilité sociale, d'autres voient difficilement comment ils pourraient échapper aux demandes qui leur sont faites par les autorités. Bref, on pourrait dire que les criminologues, mis à part une minorité, ne sont pas contre l'utilisation de la notion de dangerosité - même s'ils sont contre l'utilisation du terme - mais contre sa mauvaise utilisation dans le cadre de leurs évaluations.

La situation semble quelque peut différente en ce qui a trait à la place que les praticiens aimeraient accorder dans leurs interventions au contrôle de la dangerosité. C'est en effet par rapport à cette fonction de contrôle rattachée à leur travail que les criminologues se sentent le plus mal à l'aise. D'une part, la plupart ont intégré, de par leur formation, un modèle de pratique où ils aimeraient «faire de la relation d'aide»; d'autre part, ils se trouvent aux prises avec des conditions de pratique où ils doivent avant tout répondre à des impératifs de contrôle, ce qui n'est pas sans leur causer des conflits idéologiques vécus d'une façon plus ou moins intense selon chacun. La notion de dangerosité rejoint donc des interrogations plus profondes sur le modèle de pratique que voudraient se donner les criminologues comme groupe professionnel et sur les orientations effectives que le système pénal donne à leur pratique.

\section{CONCLUSION}

À partir des critiques soulevées dans la littérature et après avoir vu différentes dimensions de l'utilisation de la notion de dangerosité, nous allons maintenant aborder quelques pistes de réflexion qui, nous le souhaitons, pourront faire avancer le débat. 
Nous avons vu qu'une bonne partie de nos interviewés considèrent que l'évaluation de la dangerosité soulève un certain nombre de problèmes. Nous avons vu également qu'à toutes les étapes du processus pénal, on demande aux criminologues de se prononcer sur cette question et d'en tenir compte dans leurs diverses interventions. Nous avons vu enfin que l'évaluation de la dangerosité est un processus de reconstruction où interviennent des considérations aussi bien professionnelles et sociales que politiques. À la suite de ces observations, peut-on prendre un certain recul par rapport à la pratique criminologique?

Deux niveaux d'interrogation nous paraissent particulièrement pertinents. Le premier a trait à la compétence des criminologues à se prononcer sur la dangerosité. Compte tenu des difficultés actuelles associées à la prédiction et des conséquences que cela peut avoir pour la clientèle, peut-on et doit-on continuer à se prononcer sur la dangerosité? Les points de vue diffèrent sur cette question. Certains estiment qu'il serait préférable au plan éthique de s'abstenir de faire de telles évaluations, du moins jusqu'à ce qu'on ait découvert des instruments plus valables. D'autres pensent qu'il vaut mieux continuer à se prononcer, quitte à utiliser des critères plus factuels (âge, nature et date du dernier délit, nombre d'antécédents de violence, nombre et durée des incarcérations antérieures, etc.), ou à offrir de meilleures garanties légales (droit de consulter son dossier, droit d'être représenté par un ami ou un professionnel). Ces solutions visent à améliorer les évaluations et le sort des individus concernés, et à ce titre, ne sont pas négligeables. Cependant, les problèmes liés à l'évaluation de la dangerosité n'épuisent pas toutes les facettes de la question. Ceci nous amène au deuxième niveau d'interrogation.

Quelles sont les conséquences, et pour la pratique criminologique et pour la politique pénale, de recourir à la notion de dangerosité? Concernant le premier aspect, en mettant l'accent sur la dangerosité, la pratique ne risque-t-elle pas d'être axée sur un modèle de contrôle? Ceci pose la question de savoir quel type de pratique désirent se donner les criminologues, et celle de l'indépendance qu'ils peuvent avoir par rapport aux demandes du système pénal. Concernant le second aspect, le recours à la notion de dangerosité pose déjà plusieurs problèmes comme par exemple, les sentences-vie éligibles à 25 ans et les injustices pouvant découler de l'incertitude des prédictions. De plus, le recours à cette notion soulève des questions plus fondamentales quant à l'orientation actuelle du système pénal. Outre qu'elle met l'accent sur un certain type de «dangerosité», cela ne risque-t-il pas de perpétuer l'image d'une «délinquance 
dangereuse» et de justifier une intervention pénale qui autrement, pourrait apparaître plus discutable?

Si l'on admet toutes ces questions, l'on constatera rapidement qu'il n'y a pas de solution miracle, mais peut-être une occasion de repenser la pratique criminologique.

\section{RÉFÉRENCES}

ARSENAULT, C. (1981), "La libération conditionnelle : le point de vue des acteurs", Criminologie, XIV, $\mathrm{n}^{\circ} 2$, p. 41-60.

BOTTOMS, A.E. (1977), «Reflections on the Renaissance of Dangerousness", Howard Journal of Penology and Crime Prevention, 16, $\mathrm{n}^{\prime \prime}$ 2, p. 70-96.

Commission de réforme du droit au Canada (1976), Principes directeurs et mesures non sentencielles dans le processus pénal. Ottawa, Information Canada.

DEBUYST, Ch. (1977), «Le concept de dangerosité et un de ses éléments constitutifs : la personnal ité (criminelle)", Déviance et société, 1, n" 4, p. 363-387.

DEBUYST, Ch. (1981), «Le concept de personnalité dangereuse considéré comme expression d'un point de vue", in Ch. Debuyst (édit.), Dangerosité et justice pénale. Ambiguité d'une pratique (pp. 19-34), Genève, Médecine et Hygiène.

DOZOIS, J., M. LALONDE, J. POUPART, (1981), «La dangerosité : un dilemme sans issue? Réflexions à partir d'une recherche en cours", Déviance et société, $\mathrm{V}$, p. $383-401$.

FRANÇOIS, J. (1979), "La dangerosité en milieu pénitentiaire», in Ch. Debuyst (édit.) : Dangerosité et justice pénale. Ambiguité d'une partique (pp. 83-105), Genève, Médecine et Hygiène.

GEIS, G., J. MONAHAN, (1976), "Controlling "dangerous" People", Annals of the American Academy of Political and Social Science, 423, p. 142-151.

GOFFMAN, E. (1968), Asiles, Paris, Minuit.

LANDREVILLE, P., M. PETRUNIK, (1981), "Le «délinquant dangereux" dans les législations nord-américaines", in Ch. Debuyst (édit.) : Dangerosité et justice pénale. Ambiguité d'une pratique, Genève, Médecine et Hygiène, pp. 207-229.

MEGARGEE, E.I. (1976), "The Prediction of Dangerous Behavior", Criminal Justice and Behavior, 3, n" 1, p. 3-22.

MONAHAN, J. (1976), "The Prevention of Violence", in J. Monahan (édit.), Community Mental Health and the Criminal Justice System. New York, Pergamon press, pp. 13-35.

MONAHAN, J. (1978). "The Prediction of Violent Behavior : a Methodological Approach Critique and Prospectus", in U.S. National Academy of Sciences, Deterrence and Incapacitation, Washington, pp. 244-269.

MONAHAN, J. (1981), Predicting Violent Behavior. An Assessment of Clinical Techniques, London, Sage.

MONTANDON, C. (1979), "Actualités bibliographiques : la dangerosité, revue de la littérature anglo-saxonne». Déviance et société, 3, n"1, p. 89-104.

MORRIS, N. (1974), The Future of Imprisonment, Chicago, University of Chicago Press. 
PFOHL, S.J. (1978), Predicting Dangerousness : the Social Construction of Psychiatric Reality, Massachusetts, D.C. Heath.

POUPART, J.. J. DOZOIS, M. LALONDE, (1982), "L'expertise de la dangerosité», Criminologie, XV, n" 4, p. 7-25.

SHAH, S.A. (1977), "Dangerousness : some Definitional, Conceptual and Public Policy Issues", in B.D. Sales (Édit.), Perspectives in Law and Psychology. The Criminal Justice System, New York, Plenum Press, pp. 91-121.

VON HIRSH, A. (1972. "Prediction of Criminal Conduct and Preventive Confinement of Convicted Persons", Buffalo Law Review, 21, n" 3, p. 717-758. 